\title{
Consolidation of multifeature items in visual working memory: Central capacity requirements for visual consolidation
}

\author{
Biljana Stevanovski • Pierre Jolicœur
}

Published online: 15 February 2011

(C) Psychonomic Society, Inc. 2011

\begin{abstract}
In the present study, we examined whether greater attentional resources are required for consolidating two features (e.g., color and orientation) than for consolidating one feature (e.g., color) in visual working memory (WM). We used a dual-task procedure: Subjects performed a WM task and a secondary probe task, sometimes concurrently. In the WM task, subjects decided whether two displays (containing one to four objects composed of one or two features) were the same or different. In the probe task, subjects made a speeded discrimination response to a tone. Performance in both tasks was impaired when they were performed concurrently; however, performance costs in the tone task were not greater for multi- than for single-feature conditions (when the orientation and conjunction conditions were considered). Results suggested that equivalent attentional resources were necessary for consolidation of singleorientation or multifeature items.
\end{abstract}

Keywords Visual working memory · Attentional resources $\cdot$ Consolidation $\cdot$ Features $\cdot$ Conjunctions

Visual working memory (WM) refers to the memory store that is used to maintain visual information, including information about features (e.g., color and shape), for several seconds. In the present work, we examined whether there are greater attentional resource requirements for

B. Stevanovski $(\bowtie)$

Department of Psychology, University of New Brunswick, PO Box 4400, Fredericton, New Brunswick E3B 5A3, Canada e-mail: biljana.stevanovski@gmail.com

P. Jolicœur

Département de Psychologie, Université de Montréal,

Montréal, QC, Canada consolidating two features (e.g., color and orientation) in visual WM than for one feature (e.g., color). That is, are more attentional resources recruited to consolidate a red oriented line and a green oriented line than would be necessary to consolidate two black oriented lines? The present study examined this question using a dual-task procedure in which subjects performed a visual WM task and a secondary probe task, sometimes concurrently.

Visual WM has been studied effectively using a change detection procedure (e.g., Morey \& Cowan, 2004; Treisman \& Zhang, 2006; Vogel, Woodman, \& Luck, 2001). In this procedure, a study display containing a number of visual items is briefly presented. Following a blank retention interval, a test display is presented, and subjects must decide whether the two displays are the same or different. The number of display items (set size) is manipulated across trials. Typically, individuals are very good at performing this task when set sizes are less than four items, but performance decreases for larger set sizes (Vogel et al., 2001).

Thus, investigations of the storage capacity of visual WM have often suggested that visual WM is limited to about four items (e.g., Vogel et al., 2001). Interestingly, researchers have also found that the storage capacity limit is the same for objects composed of more than one set of visual features, even when that item is composed of up to four features. For example, in their Experiment 14, Vogel et al. (2001) reported that performance was very good for up to four items, even when each item was composed of up to four features (e.g., gap presence or absence, size, color, and orientation). Thus, visual WM storage appears to be limited to about four "objects" composed of one or more features. Interestingly, Wood (2007) found evidence that there is also a visual WM system that is specialized for human actions. The storage capacity limit for this visual WM subsystem 
was also quite low (about two to three actions), but, importantly, storing more properties or features across those actions could also increase storage. That is, nine properties could be remembered almost as well as three properties, when those properties were distributed across three actions. Thus, it seems that visual WM (including memory for objects or actions) allows for the chunking of information such that performance is very similar for objects composed of a single feature or multiple features.

Recently, Woodman and Vogel (2008) investigated whether subjects obligatorily encode in visual WM the multiple features of an object or whether subjects can selectively encode (or consolidate) a given feature of a multifeature object. For example, if presented with a set of colored shapes, can subjects selectively encode only the color information, or is encoding of the entire "object" obligatory, such that color and shape information must also be encoded? This question has implications for previous work that has shown that items composed of multiple features are recalled as well as items composed of one feature (Vogel et al., 2001). It is possible that equivalent memory performance was found because subjects are unable to encode selectively and, thus, all features are spontaneously encoded in visual WM. This issue has been addressed using human electrophysiological evidence with a component of the event-related potential (ERP) analysis of the electroencephalogram. Klaver, Talsma, Wijers, Heinze, and Mulder (1999) observed an ERP correlate of visual short-term memory in which the voltage at posterior electrodes contralateral to the side of an encoded visual stimulus (e.g., a right-sided electrode when the encoded stimulus was in the left visual field) is more negative than the voltage at the corresponding ipsilateral electrode. This pattern has been variously called contralateral negative slow wave (Klaver et al., 1999), sustained posterior contralateral negativity (SPCN: Jolicœur, Brisson, \& Robitaille, 2008), or contralateral delay activity (Vogel \& Machizawa, 2004). This ERP has been found during stimulus presentation (Klaver et al., 1999), during multiple-object tracking (Drew \& Vogel, 2008), and during curve tracing (Lefebvre, Jolicœur, \& Dell'Acqua, 2007). We prefer to refer to this ERP pattern as SPCN because it emphasizes the very posterior scalp distribution that is characteristic of this response.

Although the SPCN has been found in other tasks, it is most frequently observed in situations in which lateralized visual stimuli are encoded in visual WM (e.g., Jolicœur et al., 2008; McCollough, Machizawa, \& Vogel, 2007). Vogel and Machizawa (2004) showed that the amplitude of the SPCN increases as the number of encoded objects increases, up to the capacity of visual WM, suggesting that the SPCN is a good index of visual WM (see also Jolicœur et al., 2008). Woodman and Vogel (2008) examined whether the SPCN was larger in amplitude when subjects needed to remember color and orientation information, relative to when subjects could ignore one feature dimension and encoded color alone or orientation alone. The SPCN was larger for the color-plus-orientation condition, relative to the color-alone condition. Thus, their data suggest that subjects can consolidate selective features of a multifeature item.

Recent work has demonstrated that visual WM is also limited by capacity limitations on consolidating information in memory. Specifically, Stevanovski and Jolicœur (2007) examined the processing capacity limitations of simple features, such as color, in visual WM. They asked subjects to perform a visual WM change detection task and a speeded tone probe task (e.g., low/high tone pitch discrimination), sometimes concurrently. The tone was presented at various stimulus onset asynchronies (SOAs) following the presentation of the visual study display (i.e., 100, 200, 400, or $1,000 \mathrm{~ms}$ following the presentation of the study display). Thus, the tasks could overlap very little (at long SOAs) or more (at short SOAs). They reasoned that if the tasks required access to the same attentional resources, then performance on one or both tasks should be impaired. If the tasks did not require access to the same resources, then performance on both tasks should be unaffected. Stevanovski and Jolicœur (2007) found that performance on both tasks was impaired when they were performed concurrently. Performance on the visual WM task was poorer when subjects performed a concurrent tone task, and this impairment increased as set size increased. Additionally, response times (RTs) in the tone task were longer when subjects performed the tone task concurrently with a memory task, and these RTs increased further as processing overlap between the tasks was increased. Interestingly, the interference effects on tone task performance did not increase as the number of to-beremembered items was increased. These interference effects were interpreted as reflecting competition for a common set of attentional mechanisms, so that memory consolidation and tone task performance were impaired when overlap among processing mechanisms required by the two tasks increased. The lack of increased interference effects on the tone task as set size increased suggests that consolidating more items does not require more attentional resources when those items are composed of a single feature (e.g., color).

In the present experiment, we were interested in examining whether the interference effects that have previously been observed (Stevanovski \& Jolicœur, 2007) would be larger when the objects to be consolidated in memory consisted of multiple features (e.g., colored oriented lines), relative to the interference observed when objects consist of a single feature (e.g., black oriented lines or colored disks). Woodman and Vogel (2008) found that subjects can selectively encode aspects of to-be-remembered items and that the SPCN is greater when subjects encode multiple features (color and orientation), relative to when subjects 
encode a single feature (color). Increasing the amount of information that is consolidated in visual WM might also tax attentional mechanisms that consolidate that information. In this case, the cost of consolidation on tone task RTs should be greater for objects composed of multiple features than for objects composed of a single feature. Alternately, the attentional mechanisms that cause dual-task interference may operate at the level of integrated objects, and if so, increasing the amount of information that needs to be consolidated in visual WM for each individual object may not require more work by these mechanisms. In this case, the cost of consolidation on tone task RTs should not differ for multifeature objects, as compared with single-feature objects.

\section{Present experiment}

We examined whether attentional limitations for consolidating information in visual WM would be greater for two features (e.g., color and orientation) than for one feature (e.g., color or orientation). That is, does doubling the number of to-beconsolidated features produce a corresponding increase in processing costs?

To investigate the capacity limitations of consolidating multifeature objects, subjects performed tasks in three experimental phases. In the dual-task phase (encode/respond tone), subjects performed both a visual WM task and a concurrent speeded tone pitch discrimination task. The visual WM task required subjects to encode objects composed of one or two features. In the single-feature conditions, subjects monitored a single feature (either color or orientation alone). In contrast, in the double-feature condition, subjects monitored both color and orientation. Performance in the dual-task phase was compared with that in two control phases (i.e., a visual WM task only phase and a tone task only phase). In the encode/no-tone condition, we established baseline WM performance for one or two features. In the ignore/respond-tone condition, subjects viewed the visual WM displays but could ignore those displays. The only task was to respond to the pitch of the tone that was presented following the visual displays. We examined interference effects by testing whether performance was poorer or less accurate in the dual-task phase than in the control conditions. Again, we were interested in whether interference in the tone task or the visual WM task would be greater when subjects monitored two features, relative to when subjects monitored one feature.

\section{Method}

\section{Subjects}

Thirty-seven undergraduates participated in two experimental sessions in exchange for pay. Data from one subject were excluded from analysis because the subject did not achieve a level of accuracy greater than $56 \%$ in the memory task, even with considerable practice. Data from another subject were incomplete due to computer malfunction. Thus, data from 35 subjects were analyzed. Subjects ranged in age from 18 to 33 years, $(M=21.0)$, and all reported normal or corrected-to-normal vision.

\section{Stimuli}

Visual stimuli were viewed from a distance of $54 \mathrm{~cm}$ and were displayed using Micro Experimental Laboratory software (Schneider, 1988). Stimuli were presented within an area that subtended $10.8^{\circ}$ in visual height and width at the display center. This area was subdivided into an invisible $5 \times 5$ grid, yielding 25 possible locations. Locations were chosen randomly on each trial and were presented so that there were $2.5^{\circ}$ between stimuli, center to center, for stimuli in adjacent locations (horizontally or vertically).

Colored disks subtended $0.9^{\circ}$ of visual angle and were presented in blue, green, yellow, or red. Line stimuli subtended $0.25^{\circ}$ of visual angle in width and $1.1^{\circ}$ of visual angle in height and were presented in black, blue, green, yellow, or red. The orientation of the line stimuli was randomly chosen, with replacement, from orientations of $0^{\circ}, 45^{\circ}, 90^{\circ}$, and $135^{\circ}$.

Subjects performed a four-alternative tone pitch discrimination (4 AD) task. Tones were low (200 Hz), medium-low (440 Hz), medium-high $(968 \mathrm{~Hz})$, or high $(2130 \mathrm{~Hz})$ in pitch. Subjects responded to the tones by pressing the "C," "D," "W," and "Q" keys, using the index, middle, ring, and little fingers of their left hand, to make low, medium-low, medium-high, and high tone pitch discrimination responses, respectively.

Subjects pressed the " $<$ " and " $>$ " keys, using the index and middle fingers of their right hand, to make same and different judgments, respectively, for the memory task. In the ignore/respond-tone phase, a cross (composed of a $45^{\circ}$ oriented line superimposed over a $135^{\circ}$ oriented line) was used in the detection task. In this task, subjects indicated the presence/absence of the cross with a press of the " $<$ " and ">" keys, respectively.

\section{Procedure}

Trials had the general structure illustrated in Fig. 1 (minor differences across phases are described in the following subsections). Two fixation symbols, displayed to the left and to the right of screen center, provided feedback for the preceding trial. A plus sign $(+)$ indexed a correct response, and a minus sign (-) indexed an incorrect response. Generally, feedback for the memory task was provided 


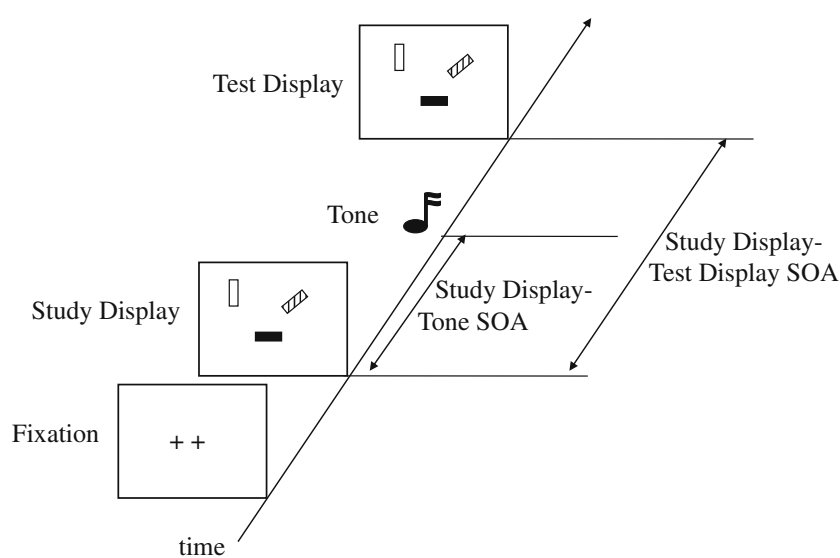

Fig. 1 Fixation symbols, which provided accuracy feedback for the preceding trial, were presented until the subject initiated the trial. A study display was presented for $100 \mathrm{~ms}$, followed by a blank retention interval. The study-display-test-display stimulus onset asynchrony (SOA) was 1,600 ms. During the blank retention interval, but not on the encode/no-tone trials, a tone was presented for $100 \mathrm{~ms}$ at a studydisplay-tone SOA of $100,200,400$, or $800 \mathrm{~ms}$. A test display was presented for $5,000 \mathrm{~ms}$ or until subjects indicated whether the two displays were the same or different

using a symbol to the right of fixation, and feedback for the tone task was provided using a symbol to the left of fixation. In phases in which the tone task was not performed, the symbol was always a plus sign. Responses were recorded using the computer keyboard. Phase order was counterbalanced across subjects, and set size and stimulus onset asynchrony (SOA) were randomized during each phase.

Encode/no-tone phase In the encode/no-tone phase, subjects performed only the visual WM task. Subjects pressed the space bar to initiate each trial. Upon offset of the fixation symbols, a study display containing a set of one to four visual items was presented for $100 \mathrm{~ms}$. Following a study-display-test-display SOA of $1,600 \mathrm{~ms}$, the test display was presented for a maximum of 5,000 ms or until a response was made. The number of items and their locations was identical in the study display and the test display, but on half of all trials, the study and test displays differed. Subjects indicated whether the displays were the same or different and were instructed to focus on the accuracy, not the speed, of their responses.

In separate phases, subjects performed the encode/no-tone phase using three types of stimuli. In the color feature phase, the visual items were red, blue, yellow, and green colored disks. In the orientation feature phase, the visual items were black lines oriented $0^{\circ}, 45^{\circ}, 90^{\circ}$, or $135^{\circ}$. In the conjunction phase, the visual items were red, blue, yellow, and green lines oriented $0^{\circ}, 45^{\circ}, 90^{\circ}$, or $135^{\circ}$.

On the trials on which the study and the test displays differed, the following changes could occur. In the color condition, the color of one disk was changed to a different color, randomly selected from the remaining three colors. In the orientation condition, the orientation of one line was changed to a different orientation, randomly selected from the remaining three orientations. In the conjunction condition, either the color or the orientation of one stimulus was changed, but never both. Changing only one feature ensured that subjects needed to remember both features to perform the memory task. Thus, on half of the different trials, the color of one line changed to a different color (orientation remained the same), whereas on the remaining half of the different trials, the orientation of one line changed (color remained the same). We note that this probe task does not encourage the binding of features into an integrated item and that some researchers (Wheeler \& Treisman, 2002) have argued that feature binding requires attentional resources. The implications of using the present type of probe, as compared with a probe that encourages binding, are considered in detail in the Discussion section.

Encode/respond-tone phase Trials in the encode/respondtone condition and the encode/no-tone condition were identical, with the following exceptions. A tone was presented for $100 \mathrm{~ms}$ at a study-display-tone SOA of 100 , 200,400 , or $800 \mathrm{~ms}$ on each trial, and subjects made a speeded pitch discrimination response to the tone. Subjects were instructed to focus on both response speed and accuracy in the tone task.

The test display was presented following the tone discrimination response. As was stated previously, the test display was presented at a study-display-test-display SOA of 1,600 ms. A tone presented at a study-display-tone SOA of $400 \mathrm{~ms}$, for example, allowed subjects $1,200 \mathrm{~ms}$ in which to execute a tone discrimination response. If subjects had not made their tone discrimination response within $1,200 \mathrm{~ms}$, the presentation of the test display was delayed until subjects made their response. The test display was presented immediately upon execution of the tone response under these circumstances, to ensure that tone task performance was not disrupted by the presentation of the test display. Previous work (Vogel et al., 2001) showed that increasing the retention interval to $4,900 \mathrm{~ms}$ does not impair performance, relative to shorter retention intervals, so increases in the retention interval should not impact memory performance.

Ignore/respond-tone phase The first visual display and the tone in the ignore/respond-tone condition were identical to those in the encode/respond-tone condition, but subjects could ignore the visual display (i.e., no task was associated with it) and performed only the tone task. In this phase, visual displays containing colored disks, black oriented lines, or colored oriented lines were randomly intermixed. 
To maintain parity with the other conditions in which subjects made a memory judgment response to the second visual display, subjects performed a detection task. The test display contained the same number of items as that presented in the ignored "study" display, but all of the items were black lines oriented $45^{\circ}$ or $135^{\circ}$. On half of all trials, one of these lines was overlaid with a line of the other orientation, thereby creating a cross. Subjects were instructed to indicate whether this cross was present or absent from the display. Again, performance in the detection task was not important, and this task was used only to maintain parity with the structure of trials in the other conditions.

\section{Design}

Subjects completed a familiarization phase prior to each phase, in which subjects performed the $4 \mathrm{AD}$ tone task. In the first session, this phase consisted of a demonstration of the tones and 24 practice tone task trials. In the second session, subjects completed 12 practice trials of the tone task alone.

In the first experimental session, subjects completed one block of 24 practice trials and two blocks of 64 experimental trials in seven phases (encode/no-tone phases for single and conjunction features; encode/respond-tone phases for single and conjunction features; ignore/respond-tone phase). In the second experimental session, subjects completed one block of 12 practice trials and two blocks of 64 experimental trials in all seven phases. In total, excluding the tone-task-only practice trials mentioned above, each subject completed 252 practice trials and 1,792 experimental trials in two experimental sessions. Each experimental session lasted 65-75 min.

\section{Results}

\section{Visual WM task performance}

Mean percentage of accuracy in memory performance was computed for each subject for each combination of feature condition (color, orientation, conjunction), tone condition (encode/respond-tone, encode/no-tone), and set size (1, 2, $3,4)$. Mean accuracy in the visual WM task is plotted in Fig. 2 as a function of these factors.

First, we conducted analyses to establish that there was a cost (i.e., a decrement in memory performance) of performing a concurrent tone task. We conducted a within-subjects analysis of variance (ANOVA) that included feature condition, tone condition, and set size. Consistent with the WM literature (e.g., Vogel et al., 2001), memory accuracy decreased as the number of items increased, $F(3,102)=$ 132.09, $M S E=33.28, p<.0001$. Additionally, performance

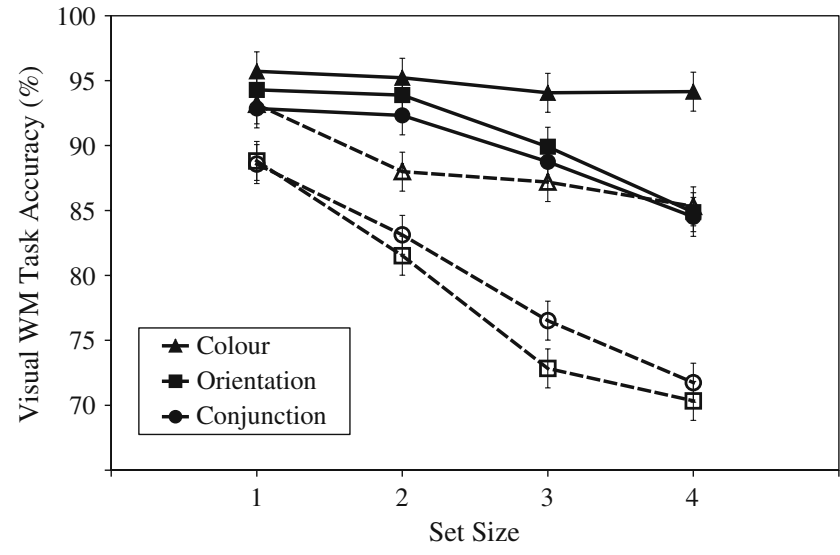

Fig. 2 Mean accuracy (in percentages) in the visual working memory (WM) task as a function of feature condition, tone condition, and set size. Solid lines correspond to the no-tone condition, and dashed lines correspond to the tone condition. The $95 \%$ within-subjects confidence interval (Loftus \& Masson, 1994) is depicted

differed across the color (91.6\%), orientation (84.6\%), and conjunction $(84.8 \%)$ conditions, $F(2,68)=120.76, M S E=$ $37.08, p<.0001$, consistent with the results of Woodman and Vogel (2008).

Of interest in the present study was whether memory performance would be affected by a secondary tone task. If both tasks rely on a common attentional resource, then performance on the memory task should be impaired by the secondary tone task. Consistent with this idea, memory accuracy was poorer when subjects performed the memory task concurrently with the tone task $(82.3 \%)$ than when it was performed alone (91.7\%), $F(1,34)=262.00, M S E=71.60$, $p<.0001$, and this effect was more pronounced as set size increased, $F(3,102)=32.21, M S E=22.93, p<.0001$. This larger decrease in memory performance in the encode/ respond-tone condition, as compared with the encode/no-tone condition, across increasing set size is consistent with the idea that both tasks rely on a common resource. The interaction between set size and feature condition was also significant, likely reflecting the slightly larger drop in performance across set size for the orientation and conjunction conditions than for the color condition, $F(6,204)=21.92, M S E=18.21$, $p<.0001$.

Of further interest was whether such memory impairments would vary for the different to-be-encoded materials. If encoding multiple features requires more attentional resources than does encoding single features, then the concurrent tone task may impair memory performance in the conjunction condition more than in the color or orientation conditions. The difference in memory performance for the encode/no-tone and encode/respond-tone conditions varied for the different feature conditions $(6.4,12.4$, and $9.6 \%$ for the color, orientation, and conjunction conditions, respectively), $F(2,68)=24.57$, $M S E=25.60, p<.0001$. The three-way interaction involving 
feature condition, set size, and tone condition was marginally significant, $F(6,204)=2.12, M S E=20.50, p<.053$. An examination of Fig. 2 suggests that this near-significant interaction may reflect the less pronounced set size $\times$ tone condition interaction for the color condition, as compared with the orientation and conjunction conditions. This pattern suggests that memory performance for color is not impaired by a concurrent task to the same degree as memory performance for orientation or for conjunctions as set size increases. Note that performance in the orientation and conjunction conditions was very similar, suggesting that the attentional resource demands of encoding a single orientation feature are similar to the demands of encoding multiple features (conjunctions).

Next, we more closely examined whether the costs to memory performance were equivalent for the single-feature conditions and whether memory impairments were greater in the conjunction condition, relative to the single-feature conditions. We conducted ANOVAs for each pairwise comparison of feature condition that treated feature condition, tone condition, and set size as within-subjects factors. If the memory impairments are greater for the conjunction condition than for the single-feature conditions, particularly when a concurrent task is performed, then this may be suggestive of larger attentional resource requirements for encoding a multifeature object, as compared with a single-feature object.

For ease of exposition, only main effects and interactions involving feature condition are considered in detail. However, the main effects of tone condition and set size, as well as the interaction between tone condition and set size, were significant for all pairs, $p s<.0001$.

The main effect of feature condition was not significant for the orientation-conjunction comparison, $F<1$, reflecting the similar memory performance for both conditions. In contrast, the main effect of feature condition was significant for the color-orientation and color-conjunction comparisons, $F(1,34)=140.16, M S E=49.58, p<.0001$, and $F(1,34)=$ 209.17, $M S E=30.97, p<.0001$, respectively, reflecting better memory performance for color than for the other conditions.

The set size $\times$ feature interaction was not significant for the orientation-conjunction comparison, $F<1.3, p>.27$, suggesting similar performance for these conditions as set size increased. This interaction was significant for the colororientation and color-conjunction comparisons, $F(3,102)=$ $39.01, M S E=17.30, p<.0001$, and $F(3,102)=30.71$, $M S E=16.13, p<.0001$, respectively. These interactions reflected a larger drop in memory performance with increasing set size for the orientation and conjunction conditions, as compared with the color condition.

The tone condition $\times$ feature condition interaction was significant for all comparisons. There was a greater cost (of a concurrent tone task) to memory performance for the orientation condition than for the color condition, $F(1,34)=$
38.88, MSE $=32.27, p<.0001$, and for the conjunction condition than for the color condition, $F(3,102)=17.53$, $M S E=21.06, p<.0002$. Interestingly, the orientationconjunction comparison revealed that the difference between tone conditions was larger for the orientation condition than for the conjunction feature condition, $F(1,34)=$ 11.20, $M S E=23.46, p<.003$. These results are particularly noteworthy, as they suggest that the cost of the concurrent tone task is lesser for the color condition than for the orientation or conjunction conditions. Additionally, these results suggest that the cost of the concurrent tone task is also less for the multifeature conjunction condition than for the single-feature orientation condition.

Finally, for the color-orientation comparison, the interaction between set size, tone condition, and feature condition was significant, $F(3,102)=3.69, M S E=22.12, p<.02$, reflecting the greater change in the set size effect when comparing the no-tone and respond-tone conditions for the orientation condition than for the color condition. This interaction was not significant for the color-conjunction and orientationconjunction comparisons, $F_{\mathrm{s}}<1.46, p \mathrm{~s}>.22$.

Visual WM task accuracy for encoding conjunctions Next, we focused only on change trials, in order to examine whether encoding features in the context of the conjunction task produced poorer memory performance than when each feature was encoded separately. This test would allow us to examine whether encoding a given feature (e.g., color) is impaired by encoding an additional feature (e.g., orientation), as compared with when just a single feature is encoded. If so, then memory performance for these features should be further impaired by the presence of an attention-demanding tone task.

First, we examined performance on only the color change trials when color features were encoded in the context of the single-feature color task and the multifeature conjunction task. We conducted an ANOVA that treated context, set size, and tone condition as within-subjects factors. Figure 3 a depicts these data as a function of these factors. (In order to prevent reporting duplicate patterns of results that were considered in previous analyses, the present sections focus only on the effects that involve context.) Notably, of the effects involving context, only the set size $\times$ context interaction was significant, $F(3,102)=2$. $96, M S E=28.10, p<.037$, potentially reflecting a slightly larger drop at larger set sizes for color memory performance in the context of the conjunction task, as compared with the single-feature color task. Thus, the results do not appear to be consistent with a larger attentional resource demand when color is encoded in the context of a multifeature conjunction condition versus a single-feature condition.

Next, we examined performance on only the orientation change trials when orientation features were encoded in the context of the single-feature orientation task and multifeature 


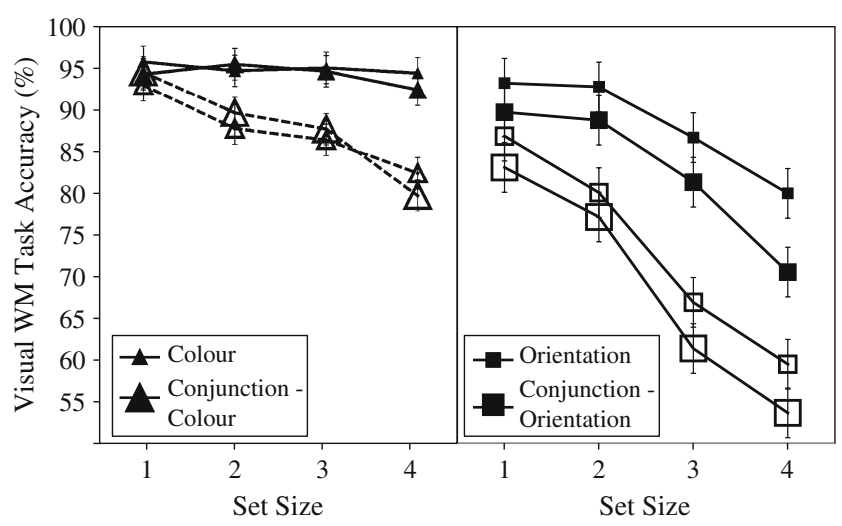

Fig. 3 Mean accuracy (in percentages) in the visual working memory (WM) task as a function of context, tone condition, and set size. Solid lines correspond to the no-tone condition, and dashed lines correspond to the tone condition. The $95 \%$ within-subjects confidence intervals (Loftus \& Masson, 1994) are depicted

conjunction task. We conducted an ANOVA that treated context, set size, and tone condition as within-subjects factors. Figure $3 b$ depicts this pattern of results. Again, as for the encoding of color information, only a single effect involving context was significant. Specifically, the main effect of context was significant, $F(1,34)=26.67, M S E=133.29, p<$ .0001 , reflecting better performance for the single-feature orientation task than for orientations in the dual-feature conjunction condition. Note, however, that this effect again did not interact with tone condition, $F<1$, as might be expected if encoding multiple features is more demanding of attentional resources than is encoding a single feature.

Visual WM task accuracy in the encode/respond-tone conditions as a function of SOA We conducted an ANOVA to examine the effects of SOA on memory, although such effects were not a focus of the experiment. This test allowed us to examine whether the timing of the concurrent tone task impacted memory performance. Mean accuracy in the visual WM task is plotted in Fig. 4 as a function of SOA and feature condition. Set size, SOA, and feature condition were treated as withinsubjects factors. (Previously, SOA was not included, because there was no such factor for the no-tone condition. In this section, we focus exclusively on effects involving SOA.) Memory accuracy decreased with increasing SOA (84.4\%, $83.2 \%, 82.2 \%$, and $79.3 \%$ for the $100-$ to $800-\mathrm{ms}$ SOAs, respectively), $F(3,102)=22.64, M S E=88.10, p<.0001$. The interpretation of this SOA effect is considered in the Discussion section. All other effects were not significant, $F \mathrm{~s}<1$.

Tone task performance

Tone discrimination response times Mean RT to make a correct tone pitch discrimination was computed for each

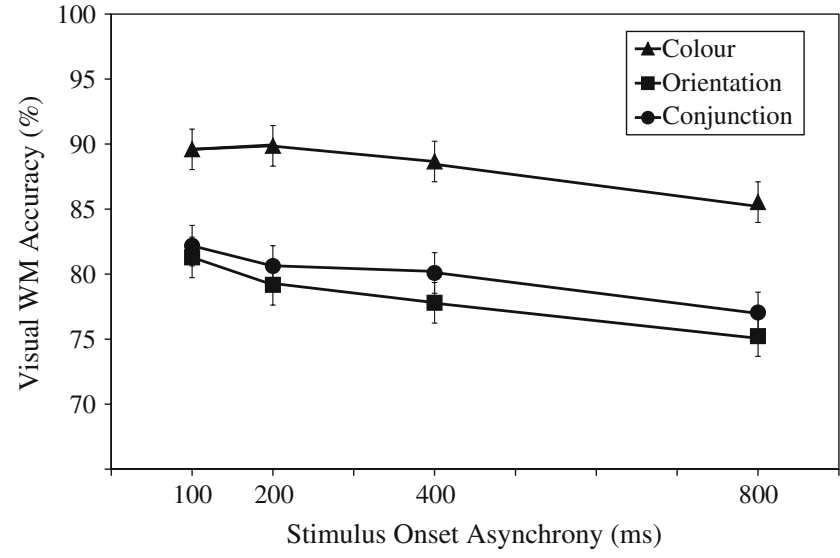

Fig. 4 Mean accuracy (in percentages) in the visual working memory (WM) task as a function of feature condition and stimulus onset asynchrony. The $95 \%$ within-subjects confidence interval (Loftus \& Masson, 1994) is depicted

subject, for each combination of tone condition (encode/ respond-tone-color, orientation, conjunction, and ignore/ respond-tone), SOA, and set size. RT data from trials on which the tone pitch was correctly identified were first submitted to a recursive outlier analysis (see Van Selst \& Jolicœur, 1994). This outlier analysis excluded $2.7 \%$ of the trials on which correct tone pitch discriminations had been made. An ANOVA was conducted, using tone condition, SOA, and set size as within-subjects factors. Tone RTs are plotted in Fig. 5 as a function of tone condition and SOA.

Of greatest interest was whether interfering effects on tone RTs were more pronounced for the conjunction condition than for the color or orientation condition. Such an effect would suggest that the attentional resource requirements for encoding visual information are greater for multiple-feature items than for single-feature items. Tone discrimination responses were made more quickly

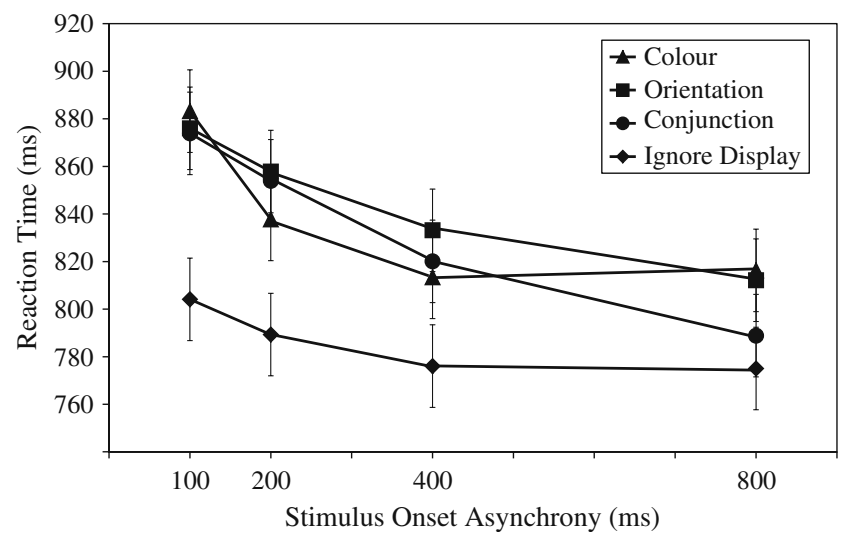

Fig. 5 Mean reaction time (in milliseconds) in the tone pitch discrimination task as a function of tone condition and stimulus onset asynchrony. The 95\% within-subjects confidence interval (Loftus \& Masson, 1994) is depicted 
when subjects could ignore the presentation of the visual display $(786 \mathrm{~ms})$ than when subjects encoded the different visual displays $(845,838$, and $834 \mathrm{~ms}$ for orientation, color, and conjunctions, respectively), $F(3,102)=5.67, M S E=$ $70,633.16, p<.002$. The effects of tone condition interacted with the effects of SOA, $F(9,306)=2.06$, $M S E=10,969.56, p<.04$, probably reflecting the fact that the effects of SOA (i.e., increase of tone discrimination RTs as a function of decreasing SOA) were less pronounced for the ignore/respond-tone condition than for the conditions in which the memory task was performed concurrently with the tone discrimination task. An examination of Fig. 5 also suggests that tone discrimination RTs for the conjunction condition were not longer than those for the single-feature conditions. Consistent with this assertion, a separate ANOVA indicated that the tone condition $\times$ SOA interaction was not significant once the ignore/respond-tone condition was excluded, $F(6,204)=1.44, M S E=11,026.26, p>.19$. The absence of a significant tone condition $\times$ SOA interaction suggests that the interfering effect of the concurrent tone task did not differ for single- and multifeature conditions, which suggests that the attentional resource requirements are similar for the different visual materials.

Tone discrimination RTs tended to increase as the number of items in the visual display increased, but this effect was not significant, $F(3,102)=1.74, M S E=20,437.09, p>.15$. RTs were significantly slowed with decreasing $\operatorname{SOA}, F(3,102)=$ 23.22, $M S E=17,683.49, p<.0001$. The interaction between the effects of set size and the effects of tone condition was not significant, $F(9,306)=1.32, M S E=14,227.91, p>.21$. Additionally, the effect of set size interacted with SOA, but this effect did not reveal a meaningful pattern of data, $F(9$, $306)=2.42, M S E=12,346.17, p<.012$. Finally, the interaction between the effects of set size, SOA, and tone condition was not significant, $F(27,918)=1.08, M S E=$ $11,494.23, p>.35$.

Tone discrimination accuracy Mean percentage for accuracy in the tone task was computed for each subject as a function of tone condition, SOA, and set size. These factors were submitted to a within-subjects ANOVA. Mean tone task accuracy is displayed in Table 1 as a function of these factors. The SOA $\times$ set size interaction was significant but did not reflect a theoretically interesting pattern, $F(9,306)=2.42$, $M S E=43.10, p<.02$. All other main effects and interactions were not significant, $F \mathrm{~s}<1.54, p \mathrm{~s}>.20$.

\section{Discussion}

We tested the hypothesis that consolidation in visual WM of multiple features would produce greater processing costs
Table 1 Mean tone task accuracy for each tone condition as a function of stimulus onset asynchrony and set size

\begin{tabular}{lllll}
\hline Set Size & 100 & 200 & 400 & 800 \\
\hline Colour & & & & \\
1 & 91.96 & 91.43 & 90.54 & 91.43 \\
2 & 91.07 & 93.04 & 90.00 & 90.54 \\
3 & 90.71 & 90.00 & 89.64 & 88.93 \\
4 & 90.00 & 90.18 & 87.86 & 92.14 \\
Orientation & & & & \\
1 & 89.29 & 87.82 & 90.71 & 91.95 \\
2 & 89.46 & 90.18 & 90.17 & 89.08 \\
3 & 90.71 & 91.24 & 88.57 & 87.63 \\
4 & 91.07 & 89.11 & 88.36 & 90.18 \\
Conjunction & & & & \\
1 & 91.07 & 91.79 & 89.29 & 91.25 \\
2 & 91.61 & 91.07 & 89.11 & 89.82 \\
3 & 90.71 & 88.75 & 91.43 & 90.18 \\
4 & 91.61 & 90.36 & 87.32 & 89.64 \\
Ignore Display & & & & \\
1 & 90.18 & 89.29 & 92.32 & 89.29 \\
2 & 91.79 & 92.14 & 90.36 & 89.11 \\
3 & 89.82 & 91.07 & 90.89 & 89.64 \\
4 & 88.75 & 92.86 & 89.64 & 92.50 \\
\hline
\end{tabular}

Note. The 95\% within-subjects confidence interval (Loftus \& Masson, 1994) is equal to $M \pm 2.37$

than would consolidation of a single feature. The results did not support this hypothesis: Memory performance in the conjunction condition was not significantly poorer than that in the orientation condition. In fact, the effect of performing the concurrent tone task produced larger costs for orientation features than for conjunctions. This result is qualified, however, by the fact that performing the memory task with color stimuli seemed to be easier than for orientation stimuli or for color-orientation conjunctions; that is, memory in the color condition was significantly better than memory in the orientation and conjunction conditions. This result is consistent with that of Woodman and Vogel (2008), who found differences in performance for color versus orientation features and conjunctions of color and orientation; they argued that this difference was observed because consolidation rates were more efficient for color than for other features or feature conjunctions. Even though performance for the conjunction condition was poorer than that for color in the present study, this does not show definitively that doubling the number of features increases the processing costs observed in the present experiments. More important, the cost to performance for the conjunction feature load was not significantly greater than the cost for orientation, suggesting that double-feature loads do not have larger processing costs. Although we have presented a 
null result, there are other results in the literature that corroborate our results (e.g., Allen, Baddeley, \& Hitch, 2006); these studies are discussed later in the Discussion section.

Why was memory performance more accurate in the color condition than in the other conditions? The superior performance for colors might be due to the high-contrast colors that were used. Alternately, it might suggest that encoding color into visual WM is not subject to processing capacity limitations, whereas encoding of orientation is subject to those limitations. This argument would predict that performance should be much better for color than for orientation stimuli, which is what was observed. There are several results that argue against this account. First, there was a set size effect in memory performance for color features. Also, set size effects (on memory performance) were more pronounced when subjects performed a concurrent tone task than when subjects performed the memory task alone. Finally, the pattern of interference on tone task performance due to performing a concurrent memory task (i.e., the SOA effect in tone RTs) was pronounced and statistically significant in the color condition, as well as in the orientation and conjunction conditions. Consistent with the idea that processing costs are no greater for two sets of features than for one set of features, the effect of SOA (on tone RTs) was not more pronounced for conjunctions than for orientation and color when subjects performed the tasks concurrently (refer to Fig. 5).

Additional analyses were conducted to determine whether there were greater attentional capacity demands when multiple features were encoded, as compared with single features. Specifically, we examined performance on change trials in the conjunction condition and compared this with performance on change trials in the single-feature conditions. Results suggested that encoding color plus another feature (see Fig. 3a) did not produce a decrement to memory performance, as compared with when color alone was encoded. Importantly, even when participants performed the tone task, there was not a large drop in memory performance for the conjunction color condition, as compared with the coloralone condition; this pattern is consistent with equivalent attentional requirements for encoding one versus two features. The corresponding analyses for the orientation feature conditions revealed a slightly different pattern of results (see Fig. 3b). Memory performance was poorer in the conjunction orientation condition than in the orientationalone condition, which might be suggestive of additional demands related to encoding a second feature. However, this effect did not interact with tone condition (whether participants performed a concurrent attentionally demanding tone task or not), which would be expected if encoding a second feature had additional attentional resource requirements. So it appears as though encoding orientation plus a second feature is more difficult than encoding orientation alone, but this difficulty is not increased in magnitude by a concurrent attentionally demanding task.

In combination, then, the results of the present experiment suggest that the processing capacity limitations that were observed in earlier experiments for single features are not greater in magnitude when the number of feature types is increased. These results suggest that "objects," or conjunctions of features, produce the same costs as single features. One possibility is that these features are encoded into visual WM as loose features and not as an "object." Wheeler and Treisman (2002) argued that each feature or property can be stored in independent feature stores (e.g., color and shape features would have separate stores) but that storing these features as a bound object requires attentional resources. Indeed, they examined whether performance is poorer when features need to be bound into an object by using a probe technique in which the features of two objects would be swapped. For example, a possible study display may contain a horizontal blue line and a red triangle. On a different trial, the test display would swap the features of two items, so that the overall features are identical but the combinations of those features have changed. Thus, the test display could contain a horizontal red line and a blue triangle. Using this probe task, a participant would be unable to perform the task correctly unless the features had been correctly bound into an object in memory. Consistent with their hypothesis, Wheeler and Treisman found that performance was poorer in a binding condition than in nonbinding conditions (similar to the probe conditions used by Vogel et al., 2001). They also suggested that binding features into an object thus requires attention. These results may suggest that equivalent processing costs were found in the present experiment for single- and double-feature loads because no binding of features had taken place (due to our use of a probe that did not require feature binding). The results of Wheeler and Treisman (see also Delvenne \& Bruyer, 2004) suggest that larger processing costs would be expected, if the probe encouraged or required binding of features into an object.

More recently, researchers (e.g., Gajewski \& Brockmole, 2006; Johnson, Hollingworth, \& Luck, 2008) have argued that feature binding does not require attention. Johnson et al. argued that Wheeler and Treisman (2002) had confounded feature and binding changes with changes in the configuration of items between study and test. Additionally, they argued that Wheeler and Treisman's test of attentional resource requirements was quite subtle (i.e., differences in performance due to a whole image probe or a single item probe). In their work, Johnson et al. asked subjects to perform a visual WM task that involved feature and binding conditions. During the retention interval, subjects performed an attentionally demanding visual 
search. They found that there was no difference in search task performance as a function of condition (binding or feature WM task). Although memory was somewhat poorer in the binding condition than in the feature condition, performance was not differentially affected by the secondary visual search task. These results suggest that processing costs for encoding multifeature and single-feature objects may not differ, even if features need to be bound in visual WM. Further work would be necessary to confirm that this is the case.

The results of the present experiment are also consistent with the results of Allen et al. (2006). They examined whether binding information in visual WM was an active process that required attentional resources or whether binding occurred automatically without the requirement of attentional resources. Allen et al. asked subjects to perform a visual WM task concurrently with a secondary task (e.g., a digit recall task or a backward-counting task). They found that performance was poorer due to the presence of a secondary task, but this did not differ when subjects needed to remember unbound features (e.g., remember color or shape) or bound features (e.g., remember color and shape). They argued that binding information in visual WM did not require additional attentional resources. Interestingly, they suggested that bound information might be more fragile and, thus, suffer in the presence of interference. In their Experiment 5, they examined whether sequential presentation of to-be-remembered information would disrupt bindings in memory more than would single features (relative to simultaneous presentation of to-be-remembered information). They reasoned that sequential presentation would be more disrupting to the fragile bindings than to single features. Consistent with this suggestion, there was little effect of serial position for the single features, but there was an effect for the combination or bindings condition.

Although SOA effects on memory performance were not originally of special interest in the present study, we found that there was an impact of the timing of the tone task. Specifically, memory performance decreased as SOA increased (i.e., as overlap between the presentation of the memory array and the tone decreased). One possible explanation for this pattern of results is that participants were trading off performance in the two tasks. According to this interpretation, participants may have focused on the memory task at short SOAs, which led to a slowing of RTs in the tone task and good performance in the memory task. As SOA increased, however, focus may have shifted to the tone task, which led to faster responses in the tone task and poorer performance in the memory task. In order to investigate this possibility, we conducted an analysis for memory performance at the 800-ms SOA; we performed a median split on RTs and then examined memory performance associated with the longer versus the shorter RTs. The trade-off account above would predict that longer RTs in the tone task should be accompanied by better memory performance and shorter RTs in the tone task should be accompanied by poorer memory performance. The results of our analyses revealed the opposite pattern of results: Better memory performance $(82.4 \%)$ was associated with shorter RTs, and poorer memory performance (76.2\%) was associated with longer RTs, $p<.0001$. Furthermore, this pattern was observed for similar analyses for each SOA. Therefore, the SOA effects observed in memory performance were not consistent with such a trade-off between performance in the two tasks.

It is not clear whether this SOA effect on visual memory performance represents a disruption of the process of consolidating information in memory, a disruption of memory maintenance, or merely memory loss due to interference or decay over time. At short SOAs, a number of accounts could explain the better memory performance. One possibility is that the tone task disrupts working memory consolidation or maintenance (or both), but sensory memory is still intact and so can be accessed to recover some of the to-be-encoded information. Another possibility is that the nascent memory trace is protected during consolidation, so that memory performance is better when the tone is presented during this time period, as compared with at a later time. At long SOAs, the memory maintenance process may be disrupted by the tone task, or there may even be some passive loss of the consolidated visual information, leading to poorer memory than at the short SOA. In these cases, the sensory trace of the visual information has faded, so memory performance is poorer than at short SOAs. Thus, the SOA effect observed in memory performance could be explained by appealing to a disruption of consolidation or maintenance or to other general aspects of memory loss over time.

However, it is not clear how the accounts above could explain the results of the median split analysis. The median split results (i.e., shorter RTs associated with better memory and longer RTs associated with poorer memory) may reflect some aspect of the consolidation process. For example, sometimes, consolidation may be quick and efficient (and sometimes less efficient). On trials on which consolidation is efficient, a strong memory trace is created, and thus this trace is more resistant to disruption by the tone task, which leads to quick responses to the tone pitch. On trials on which consolidation is less efficient, consolidation is longer in duration and may be more prone to disruption, which leads to longer RTs in the tone task and poorer memory performance.

We now consider the differences and similarities between the present study and the study described by Woodman and Vogel (2008). In their work, they argued that individuals do not obligatorily encode all aspects of a multifeature visual item; participants can encode single 
features or conjunctions. Using a backward-masking paradigm, they found that consolidation of color alone was more efficient than consolidation of orientation or conjunctions. Likewise, the results of the present study suggest that memory performance for color features is superior to that of orientation or color-orientation conjunctions. Other aspects of these studies are quite different; Woodman and Vogel found that color features alone were consolidated more efficiently than color in the context of a color-shape conjunction. In contrast, we found that color consolidated alone or in the context of a color-orientation conjunction produced similar memory performance. There are a number of possible reasons for this discrepancy, such as the procedures and metric used. Woodman and Vogel used consolidation slope (thereby examining the amount of time that it took to consolidate each item), whereas we used memory performance. In their experiments, they used a masking technique procedure in which three study items were presented for approximately $23 \mathrm{~ms}$ and were followed by a $500-\mathrm{ms}$ mask display that was presented at various delays $(35,105,140$, and $176 \mathrm{~ms})$, relative to the presentation of the study display. They argued that the masking delay at which memory performance asymptotes reflects the amount of time required for consolidation of that display. Using this technique, they found relatively short estimates of visual memory consolidation (see also Vogel, Woodman, \& Luck, 2006), which is in contrast to the relatively longer time course suggested by the SOA-linked results of the tone probe approach that was utilized in the present study. As we have suggested previously (Stevanovski \& Jolicœur, 2007), we believe that this difference is related to the stage of processing that is affected by the procedure that was used. Specifically, we believe that the backward-masking manipulation used by Woodman and Vogel has effects at an earlier stage of processing, whereas our results reflect visual WM consolidation. Support for this contention comes from the results of studies using the attentional blink (AB) paradigm, in which two targets are presented in rapid succession. Usually, identification of the second target is impaired when it closely follows the first target. This impairment is thought to reflect slowed consolidation of a second target, due to the occupation of consolidation processes by the first target (e.g., Ptito, Arnell, Jolicœur, \& MacLeod, 2008; Vogel \& Luck, 2002). Researchers have manipulated the duration of the second target in an $\mathrm{AB}$ paradigm and have found that the effects of target duration and the $\mathrm{AB}$ effect were additive (Dell'Acqua \& Jolicour, 2000). As such, we believe that we must discriminate between an early sensory encoding and a later visual memory consolidation. The early sensory encoding, as suggested by the results of Dell'Acqua and Jolicœur, is distinct from visual memory consolidation. We suggest that the results of Woodman and Vogel reflect this earlier sensory encoding (or the accrual of early sensory information), whereas the results of the present work are consistent with the later visual consolidation that occurs over a longer time course, as has also been found using the $\mathrm{AB}$ paradigm and the tone probe paradigm (Jolicœur, 1999).

Interestingly, in the present experiment, there was no interaction between study condition, SOA, and set size. Thus, the processing costs as indexed by tone RTs and the interaction between study condition and SOA did not vary as a function of set size. Thus, similar processing costs were observed for set sizes of one or more items. This invariance may be related to the spatial configuration of the visual WM display. Several studies have indicated that spatial configuration plays a special role in visual WM (e.g., Delvenne \& Bruyer, 2006; Hollingworth, 2007; Jiang, Olson, \& Chun, 2000; Woodman, Vecera, \& Luck, 2003). For example, Woodman et al. found a contribution of configural aspects of the visual display. When objects could be grouped together on the basis of Gestalt grouping principles, those objects tended to be grouped together in visual WM. That is, when attention was cued to an object, objects that could be grouped with that object were more likely to be encoded in WM than objects that were less likely to be grouped with the cued object. Jiang et al. found that disrupting spatial configuration between study and test also impacted memory performance. Further research is necessary to determine whether the absence of this interaction is related to the encoding of spatial configuration.

Author Note We would like to thank Nelson Cowan and two anonymous reviewers for their helpful comments and questions.

This work was supported by funding from the Natural Sciences and Engineering Research Council (NSERC).

\section{References}

Allen, R. J., Baddeley, A. D., \& Hitch, G. J. (2006). Is the binding of visual features in working memory resource demanding? Journal of Experimental Psychology: General, 135, 298-313. doi:10.1037/0096-3445.135.2.298

Dell'Acqua, R., \& Jolicœur, P. (2000). Visual encoding of patterns is subject to dual-task interference. Memory \& Cognition, 28, 184 191.

Delvenne, J.-F., \& Bruyer, R. (2004). Does visual short-term memory store bound features? Visual Cognition, 11, 1-27. doi:10.1080/ 13506280344000167

Delvenne, J.-F., \& Bruyer, R. (2006). A configural effect in visual short-term memory for features from different parts of an object. The Quarterly Journal of Experimental Psychology, 59, 15671580 .

Drew, T., \& Vogel, E. K. (2008). Neural measures of individual differences in selecting and tracking multiple moving objects. The Journal of Neuroscience, 28, 4183-4191. doi:10.1523/ JNEUROSCI.0556-08.2008 
Gajewski, D. A., \& Brockmole, J. R. (2006). Feature bindings endure without attention: Evidence from an explicit recall task. Psychonomic Bulletin \& Review, 13, 581-587.

Hollingworth, A. (2007). Object-position binding in visual memory for natural scenes and object arrays. Journal of Experimental Psychology: Human Perception and Performance, 33, 31-47. doi:10.1037/0096-1523.33.1.31

Jiang, Y., Olson, I. R., \& Chun, M. M. (2000). Organization of visual short-term memory. Journal of Experimental Psychology. Learning, Memory, and Cognition, 26, 683-702. doi:10.1037/02787393.26 .3 .683

Johnson, J. S., Hollingworth, A., \& Luck, S. J. (2008). The role of attention in the maintenance of feature bindings in visual short-term memory. Journal of Experimental Psychology: Human Perception and Performance, 34, 41-55. doi:10.1037/0096-1523.34.1.41

Jolicœur, P. (1999). Concurrent response selection demands modulate the attentional blink. Journal of Experimental Psychology: Human Perception and Performance, 25, 1097-1113. doi:10.1037/0096-1523.25.4.1097

Jolicœur, P., Brisson, B., \& Robitaille, N. (2008). Dissociation of the $\mathrm{N} 2 \mathrm{pc}$ and sustained posterior contralateral negativity in a choice response task. Brain Research, 1215, 160-172. doi:10.1016/j. brainres.2008.03.059

Klaver, P., Talsma, D., Wijers, A. A., Heinze, H.-J., \& Mulder, G. (1999). An event-related brain potential correlate of visual short-term memory. NeuroReport, 10, 2001-2005. doi:10.1097/00001756199907130-00002

Lefebvre, C., Jolicœur, P., \& Dell'Acqua, R. (2007). Cortical activity associated with curve tracing in humans: Bridging the gap with monkey electrophysiology. Poster session presented at the annual meeting of Object Perception, Attention, and Memory, Long Beach, CA.

Loftus, G. R., \& Masson, M. E. J. (1994). Using confidence intervals in within-subject designs. Psychonomic Bulletin \& Review, 1, 476-490.

McCollough, A. W., Machizawa, M. G., \& Vogel, E. K. (2007). Electrophysiological measures of maintaining representations in visual working memory. Cortex, 43, 77-94. doi:10.1016/S00109452(08)70447-7

Morey, C. C., \& Cowan, N. (2004). When visual and verbal memories compete: Evidence of cross-domain limits in working memory. Psychonomic Bulletin \& Review, 11, 296-301.
Ptito, A., Arnell, K., Jolicœur, P., \& MacLeod, J. (2008). Intramodal and crossmodal processing delays in the attentional blink revealed by event-related potentials. Psychophysiology, 45, 794-803.

Schneider, W. (1988). Micro Experimental Laboratory: An integrated system for IBM-PC compatibles. Behavior Research Methods, Instruments, \& Computers, 20, 206-217.

Stevanovski, B., \& Jolicœur, P. (2007). Visual short-term memory: Central capacity limitations in short-term consolidation. Visual Cognition, 15, 532-563. doi:10.1080/13506280600871917

Treisman, A., \& Zhang, W. (2006). Location and binding in visual working memory. Memory \& Cognition, 34, 1704-1719.

Van Selst, M., \& Jolicœur, P. (1994). A solution to the effect of sample size on outlier elimination. The Quarterly Journal of Experimental Psychology, 47A, 631-650.

Vogel, E. K., \& Luck, S. J. (2002). Delayed working memory consolidation during the attentional blink. Psychonomic Bulletin \& Review, 9, 739-743.

Vogel, E. K., \& Machizawa, M. G. (2004). Neural activity predicts individual differences in visual working memory capacity. Nature, 428, 748-751. doi:10.1038/nature02447

Vogel, E. K., Woodman, G. F., \& Luck, S. J. (2001). Storage of features, conjunctions, and objects in visual working memory. Journal of Experimental Psychology: Human Perception and Performance, 27, 92-114. doi:10.1037/0096-1523.27.1.92

Vogel, E. K., Woodman, G. F., \& Luck, S. J. (2006). The time course of consolidation in visual working memory. Journal of Experimental Psychology: Human Perception and Performance, 32, 1436-1451. doi:10.1037/0096-1523.32.6.1436

Wheeler, M., \& Treisman, A. (2002). Binding in short-term visual memory. Journal of Experimental Psychology: General, 131, 4864. doi:10.1037/0096-3445.131.1.48

Wood, J. N. (2007). Visual working memory for observed actions. Journal of Experimental Psychology: General, 136, 639-652. doi:10.1037/0096-3445.136.4.639

Woodman, G. F., Vecera, S. P., \& Luck, S. J. (2003). Perceptual organization influences visual working memory. Psychonomic Bulletin \& Review, 10, 80-87.

Woodman, G. F., \& Vogel, E. K. (2008). Selective storage and maintenance of an object's features in visual working memory. Psychonomic Bulletin \& Review, 15, 223-229. doi:10.3758/ PBR.15.1.223 